\title{
Physics of electro-thermal effects in ESD protection devices
}

\author{
Benno Krabbenborg, Reinier Beltman, Philip Wolbert and Ton Mouthaan \\ MESA Research Institute, University of Twente, P.O. Box 217, 7500 AE Enschede, The \\ Netherlands
}

(Received January 6, 1992; accepted in revised form May 27, 1992)

\section{Summary}

Damage in ESD protection devices can be caused by high local temperatures resulting from heat generation by an ESD pulse. In order to obtain physical insight into the process that leads to permanent damage, device simulations of coupled therinal and electrical behaviour have been performed. Additional to the potential and the electron and hole concentrations the lattice temperature is solved as a variable. Simulations of ESD pulses (forward bias) applied to a diode have been performed. The discharge mechanism could be visuaiised by using the coupled thermal/ electrical model. Locations with considerable temperature rise that eventually lead to damage can be extracted from the calculated temperature distributions. Protection devices with optimum electrical and thermal characteristics can be designed by adjusting doping profiles and layout parameters. The buried layer of the protection device does not contribute in conducting current at high current levels. Therefore the buried layer is not functional in diodes that are subjected to ESD in forward bias. Measurements determining the ESD vulnerability of protection devices with and without buried layer confirm this fact.

\section{Introduction}

Thermal related failures like silicon melting and contact spiking are evident in both MOS and Bipolar devices that are subjected to ESD.

In the past some models have been developed to calculate both thermal and electrical behaviour $[1,2]$. These models were more or less based on a circuit level approach. On device level, $1 \mathrm{D}$ electrical/thermal device simulations have been performed $[3,8]$.

In order to obtain physical insight in the processes that lead to permanent damage the coupling between the thermal and electrical domain in the calculations should be complete. This can be achieved by using device simulation of fully coupled thermal and electrical behaviour. A rigourous thermodynamic treatment of heat generation and conduction in semiconductor device modelling [6] is implemented in the program TRENDY. 2D simulation is preferred above 1D simulation because especially temperature- and current density dis- 
tributions are influenced by the existence of a second dimension. These quantities are very important in analysis of ESD mechanisms.

Not only physical insight can be obtained, but 2D device simulation can also be used in designing protection devices. One can experiment by adjusting doping profiles and layouts to optimize thermal and electrical characteristics of the device before processing.

Section 2 describes the thermo-electric model used in the simulator. (This model does not include hot carrier effects.) Section 3 deals with the simulated protection device. Section 4 presents the simulation results and Section 5 presents some measurement results. The conclusions are given in Section 6.

\section{The thermal-electrical device simulator TRENDY}

Simulations of thermal and electrical behaviour of an ESD protection diode (forward biased) have been performed. This was done with the aid of the 2D process/device simulator TRENDY $[4,5,9]$. This simulator contains a fully coupled thermal/electrical model [6]. The classical set of isothermal continuity equations has been extended with a continuity equation for the thermal energy. This way not only the potential and the carrier concentrations are solved, but the lattice temperature is solved as an additional variable. Moreover an additional driving force $\nabla_{T}$ is introduced in the current equations for electrons and holes. The classical current equations are supplemented by a term proportional to this additional driving force. This results in a model with the following continuity equations. All the symbols have their usual meaning.

Poisson equation:

$\nabla \cdot(\epsilon \nabla \psi)=-q\left(p-n+N_{D}-N_{A}\right)$

Electron current continuity:

$\frac{\partial n}{\partial t}-\frac{1}{q} \nabla \cdot J_{n}=-R$

Hole current continuity:

$\frac{\partial p}{\partial t}+\frac{1}{q} \nabla \cdot J_{p}=-R$

Heat energy continuity:

$C \frac{\partial T}{\partial t}+\nabla \cdot \mathbf{J}_{Q}=H$

$C$ presents the heat capacity. The temperature dependence of this factor is fairly weak [9] and in TRENDY it has been assumed constant. The terms $R$ 
and $H$ represent the net recombination rate and the heat generation respectively. For the currents $J_{n}, J_{p}$ and $J_{Q}$ the following equations are used:

Electron current:

$\mathrm{J}_{n}=q n \mu_{n} \mathbf{E}_{n}+q D_{n} \nabla n+q n D_{n}^{T} \nabla T$

Hole current:

$\mathrm{J}_{p}=q p \mu_{p} \mathbf{E}_{p}-q D_{p} \nabla p-q p D_{p}^{T} \nabla T$

Heat energy flow:

$J_{Q}=-K \nabla T$

The factor $K$ represents the (temperature dependent) thermal conductivity. The thermal diffusion coefficients are given by the following equations [11].

Thermal diffusion coefficients:

$D_{n}^{T}=\frac{r-3 / 2}{T} D_{n}$

$D_{p}^{T}=\frac{r-3 / 2}{T} D_{p}$

The value of the parameter $r$ varies between 2 and 4. The value of $r$ depends on temperature, doping level, etc. In the case of pure lattice scattering, $r$ is equal to 2. As impurity scattering becomes more dominant, $r$ tends towards 4 . The maximum value of the thermal diffusion coefficient is about five times the minimum value (as was already mentioned by Selberherr [10]).

The heat generation term $H$ consists of three parts: the Joule heat, the Thomson heat and the recombination heat.

Joule heat generation:

$E_{\text {joule }}=\frac{\mathbf{J}_{n}^{2}}{\sigma_{n}}+\frac{\mathbf{J}_{p}^{2}}{\sigma_{p}}$

Note that the Joule heat term is always positive and that it is not equal to E.J. The Joule heat generation is the energy transfer of the carriers to the lattice as a result of the silicon resistivity.

Thomson heat generation:

$E_{\text {thom }}=T\left[\nabla P_{n} \cdot J_{n}-\nabla P_{p} \cdot J_{p}\right]$

Here $P_{p}$ and $P_{n}$ are the (absolute values) of the thermo-electric powers for holes and electrons respectively. The total thermo-electric power $P_{\text {tot }}$ gives the relation between the electric field $\mathbf{E}$ and a gradient in $T$ under condition of zero current. 
$\mathbf{E}=P_{\text {tot }} \nabla T$

The total thermo-electric power is defined by eqn. (13) [7]. For a non-degenerate semiconductor with a mean free time between collisions $t \sim|\mathbf{E}|^{-s}$ the total thermo-electric power is given by:

$$
P_{\mathrm{tot}}=-\frac{k}{q}\left[\frac{n \mu_{n}\left\{\frac{5}{2}-s+\ln \left(\frac{N_{\mathrm{c}}}{n}\right)\right\}-p \mu_{p}\left\{\frac{5}{2}-s+\ln \left(\frac{N_{\mathrm{v}}}{p}\right)\right\}}{n \mu_{n}+p \mu_{p}}\right]
$$

where $N_{\mathrm{c}}$ and $N_{\mathrm{v}}$ are the effective densities of state at the conduction- and valence band edges respectively. $P_{\text {tot }}=P_{p}-P_{n}$ with $P_{p}$ and $P_{n}$ the (absolute values of the) thermo-electric powers for holes and electrons respectively. This way $P_{p}$ and $P_{n}$ are given by the following equation.

$P_{n}=\frac{k}{q}\left[\frac{n \mu_{n}\left\{\frac{5}{2}-s+\ln \left(\frac{N_{\mathrm{c}}}{n}\right)\right\}}{n \mu_{n}+p \mu_{p}}\right]$

and

$P_{p}=k\left[\frac{p \mu_{p}\left\{\frac{5}{2}-s+\ln \left(\frac{N_{v}}{p}\right)\right\}}{n \mu_{n}+p \mu_{p}}\right]$

The Thomson heat generation corresponds with the heat generation that occurs when carriers move through a region with a gradient in the thermoelectric power $P_{c}(c=n, p)$. Thomson heat can be positive as well as negative. It can be interpreted the following way. The carriers have a certain amount of thermal energy $q P_{c} T(c=n, p)$. This thermal energy changes when $P_{c}$ changes. So by moving to a region with a different $P_{c}$ they lose thermal energy to the lattice, or gain it from the lattice. The Peltier heat is embodied in this term. The positive sign of the first term of (11) is a result of the fact that the direction of the electron current is opposite to the direction of the electron movement.

Recombination heat generation:

$$
E_{\mathrm{rec}}=q R\left[\phi_{p}-\phi_{n}+T\left(P_{p}+P_{n}\right)\right]
$$


This heat generation is the result of the energy transfer from electron-hole pairs to the lattice when they recombine. The energy that is transferred by each pair is the sum of their thermal energy $\left(q P_{n} T\right.$ and $\left.q P_{p} T\right)$ and their electrochemical energy $\left(q \phi_{p}\right.$ and $\left.q \phi_{n}\right)$. A negative recombination heat occurs when there is generation instead of recombination.

With the given model thermo-electric effects like heat generation, the Seebeck, Thomson and the Peltier effect can be simulated. The thermal behaviour is not only solved in silicon but also in all the other materials used, like oxide and aluminium.

\section{The simulated protection device}

The simulated device is an ESD protection diode processed by Philips Components Nijmegen (The Netherlands). This diode has been simulated before [1] with a simulator based on a (lumped element) circuit model, but these were simulations of the diode when stressed with an ESD current in reverse direction. The device structure and the doping profile are shown in Figs. 1 and 2 respectively. The distance between contact and diffusion is $1.5 \mu \mathrm{m}$. Using a current boundary condition at one of the contacts the diode was simulated when stressed with an ESD current pulse (Human Body Model) in forward bias. The height of the pulse was $1.5 \mathrm{e} 6 \mathrm{~A} / \mathrm{cm}^{2}$ that corresponds with $1.5 \mathrm{~A}$ for a $25 \mu \mathrm{m}$ device. Figure 3 shows the shape of the stress current.

\section{Simulation results}

\subsection{Current density distribution}

Regarding the current density distribution two cases should be distinguished. At low injection level most of the current flows through the buried layer. At high injection level this is not the case.

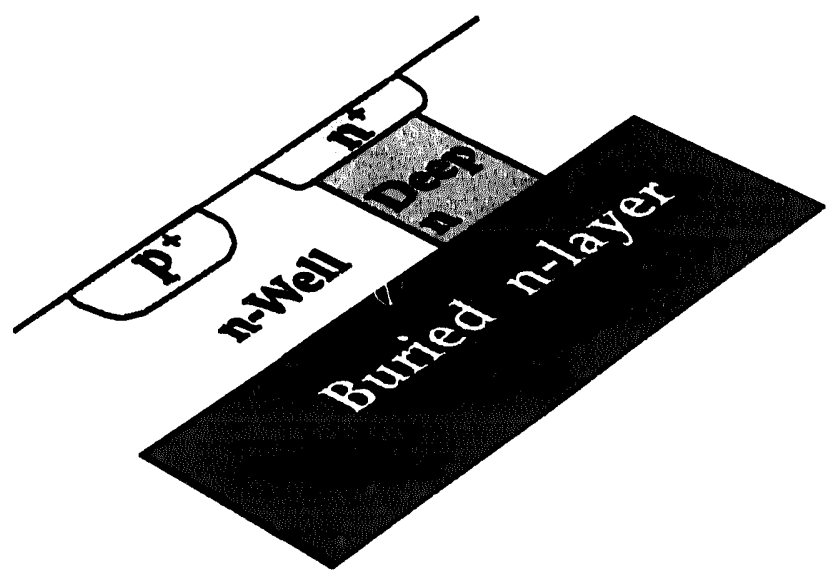

Fig. 1. Device structure. 


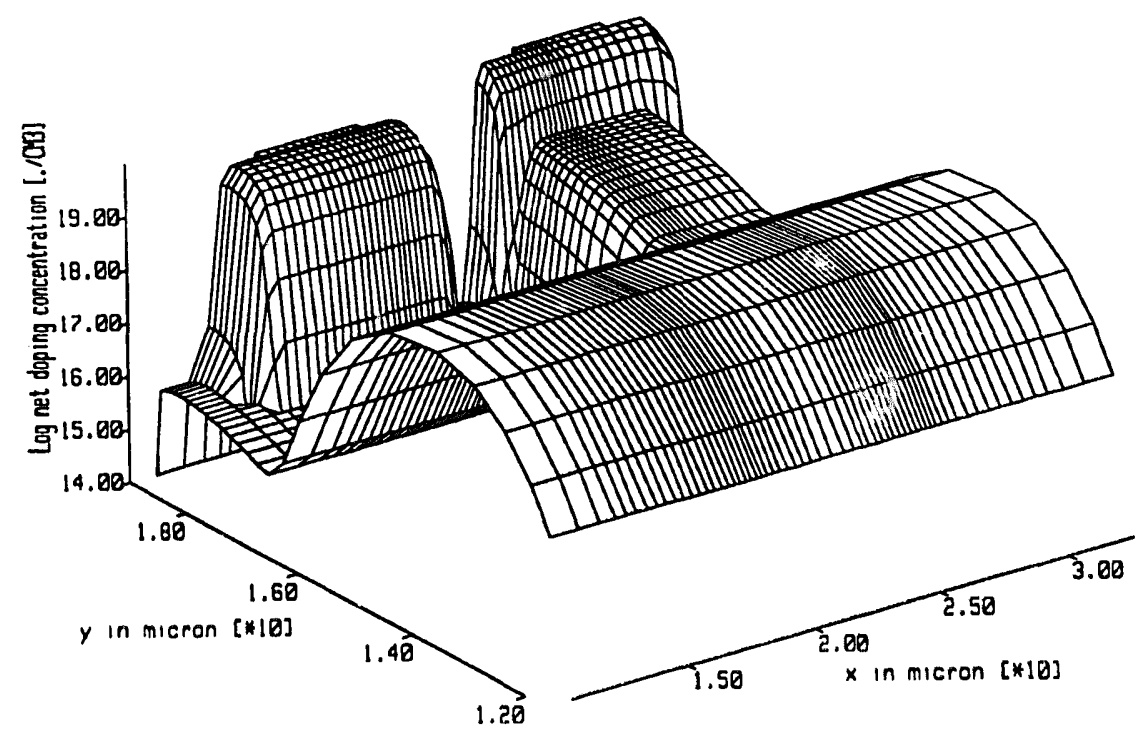

Fig. 2. Doping profile (absolute value of net doping concentration).

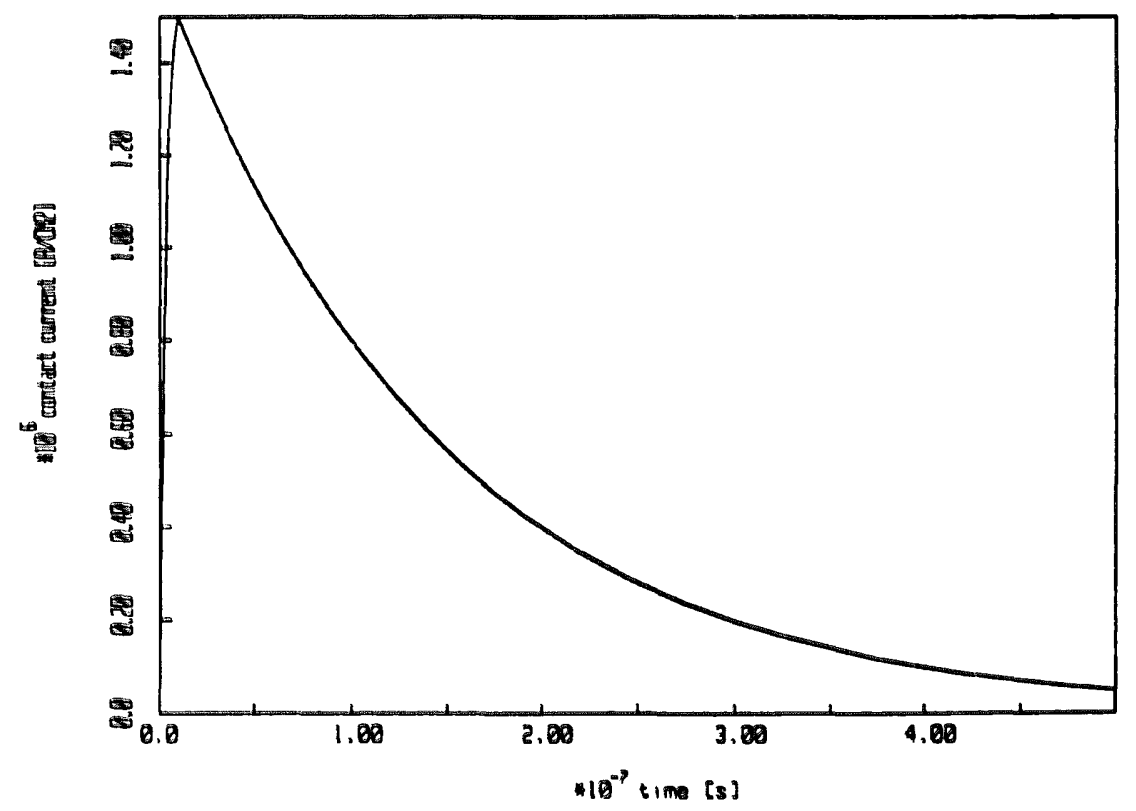

Fig. 3. Stress current versus time.

In the $n$-well, high injection of holes and electrons from the $p^{+}$and $n^{+}$ regions respectively causes the carrier concentrations to increase up to 100 or 1000 times the net doping concentration. The effect of high injection overwhelms the effect of the thermal generation of carriers. These high carrier concentrations have a positive influence on the conductivity for both electrons $\left(q n \mu_{v}\right)$ and for holes $\left(q p \mu_{p}\right)$ in the $n$-well. This effect has been simulated before [3].

Because of the high carrier concentrations, carrier-carrier scattering re- 
duces the carrier mobilities significantly. The reduction of the mobility caused by carrier-carrier scattering overwhelms the mobility reduction caused by a temperature rise. This mobility reduction has a negative effect on the conductivity. At the simulated current density leveis the electron conductivity of the $n$-well has about the same value as the electron conductivity in the buried layer. Figures 4 and 5 show the electron conductivity $q n \mu_{n}$ in the case of high and low current levels respectively.

The result is that most of the current flows directly below the surface through the $n$-well (holes arid electrons). The rest of the current (electrons), which is only a small fraction of the total current, flows through the buried layer.

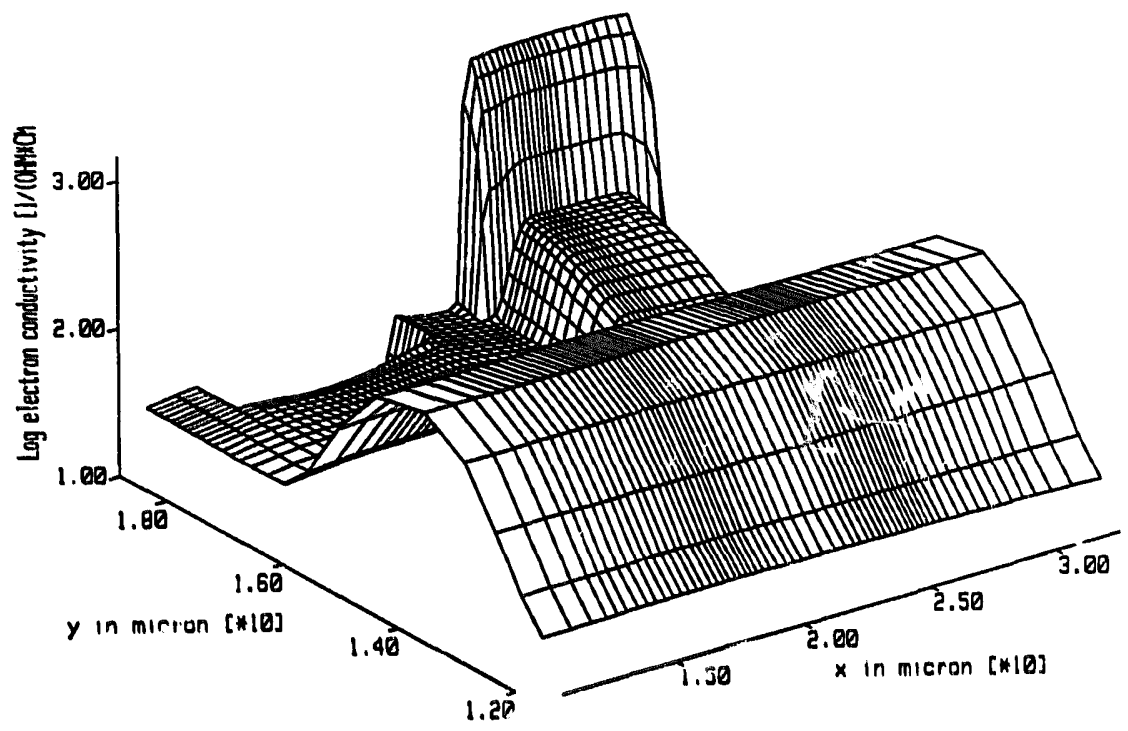

Fig. 4. Electron conductivity at low current levels.

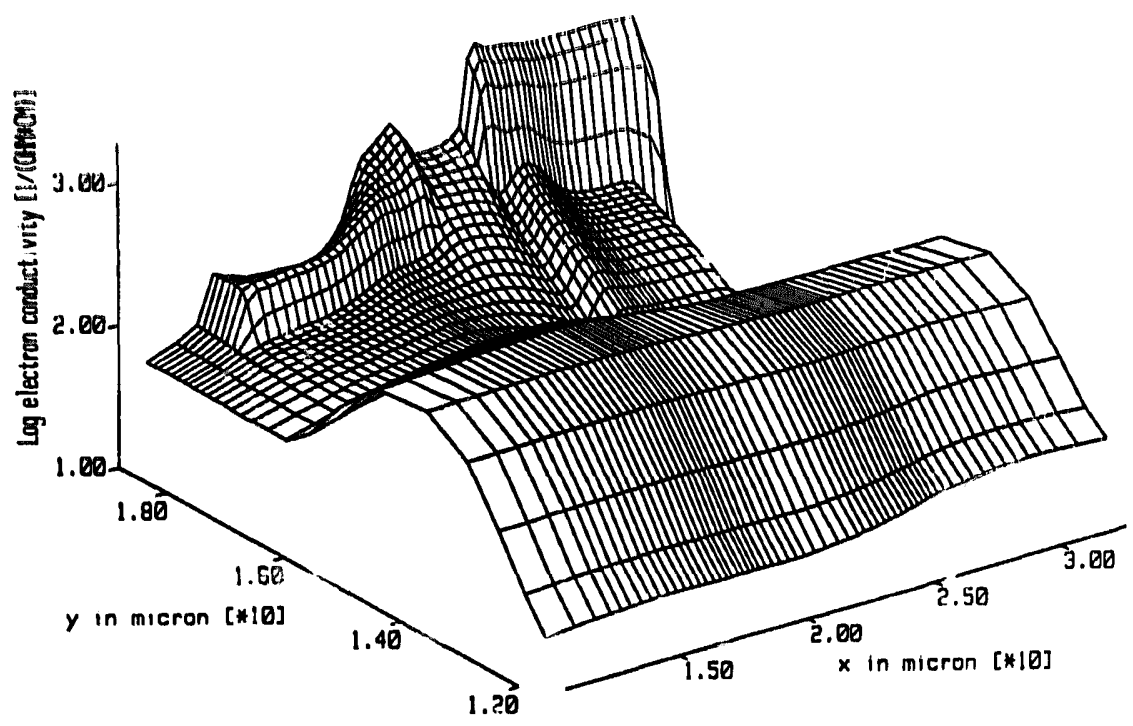

Fig. 5. Electron conductivity at high current levels. 
This effect is shown in Figs. 6 and 7. Figure 6 shows the current density distribution in the case of low level injection. The high peaks in the current density indicate the location of the contact edges. This is a two dimensional current crowding effect. It is caused by the fact that at the contact edges current can also flow into the silicon in lateral direction, (the $n^{+}$and $p^{+}$area are wider than the contact). This means that, at the edges of the contacts, the resistance to the silicon is lower than in the middle of the contacts. Figure 7 shows the case of high level injection.

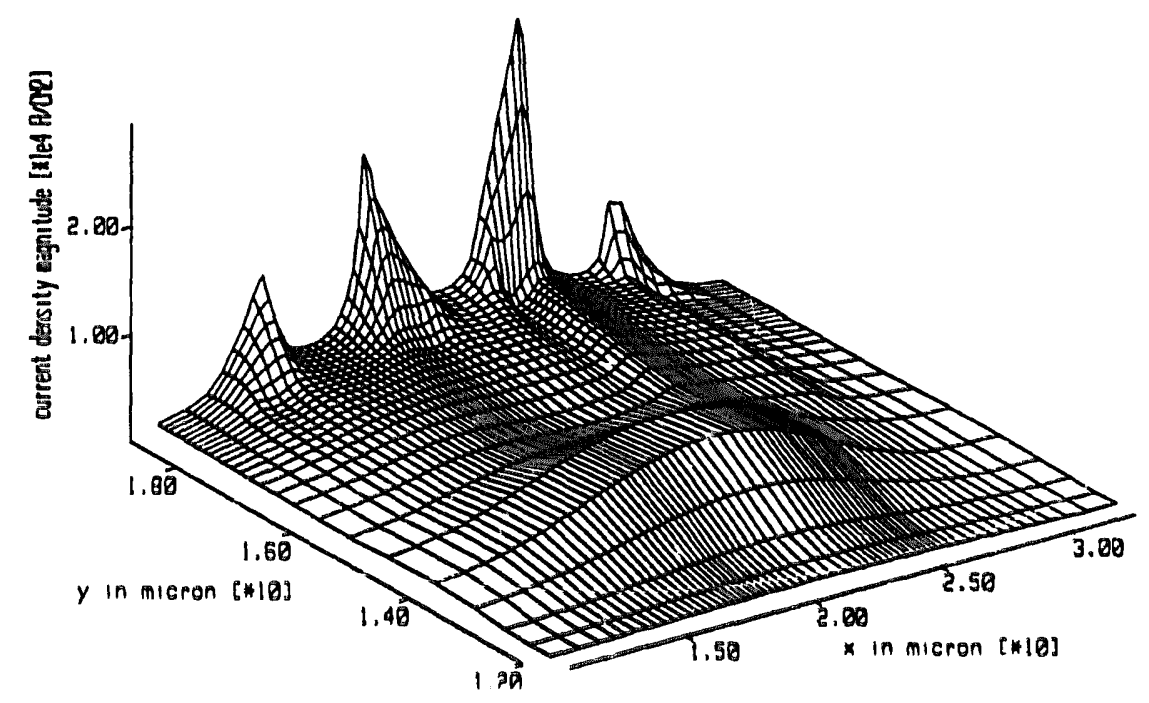

Fig. 6. Current density at low level injection.

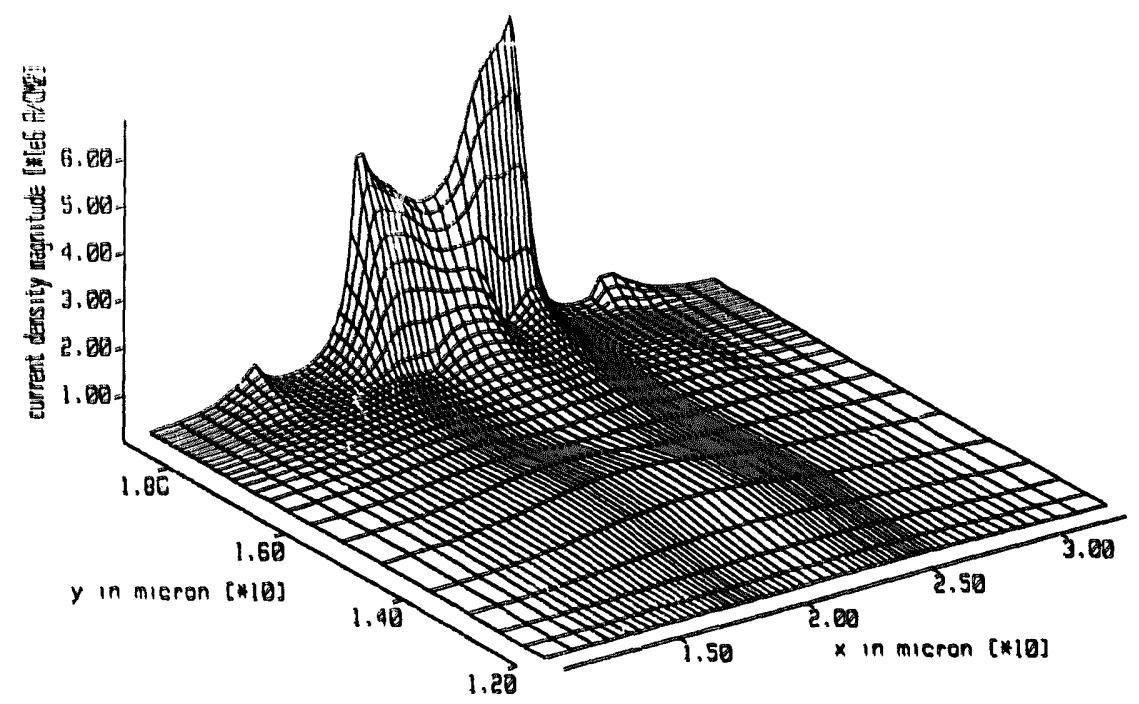

Fig. 7. Current density at high level injection. 


\subsection{Heat generation}

In the total heat generation term $H$ the Joule heat and the recombination heat play a dominant role during ESD events. Figure 8 shows the total heat generation in the diode at the time the temperature has reached the maximum value (after $75 \mathrm{~ns}$ ).

The Joule heat generation at same time is shown in Fig. 9. The highest peaks are the result of the peaks in the current densities near the contact edges.

The generation of recombination heat takes place where the recombination rate is high. Here, the highest recombination rate occurs in the corners of the $n^{+}$and $p^{+}$regions. Figure 10 shows the recombination heat generation at the time the temperature rise has reached its maximum. The peak is also visible as the highest peak in the total heat generation in Fig. 8 . The influence of the

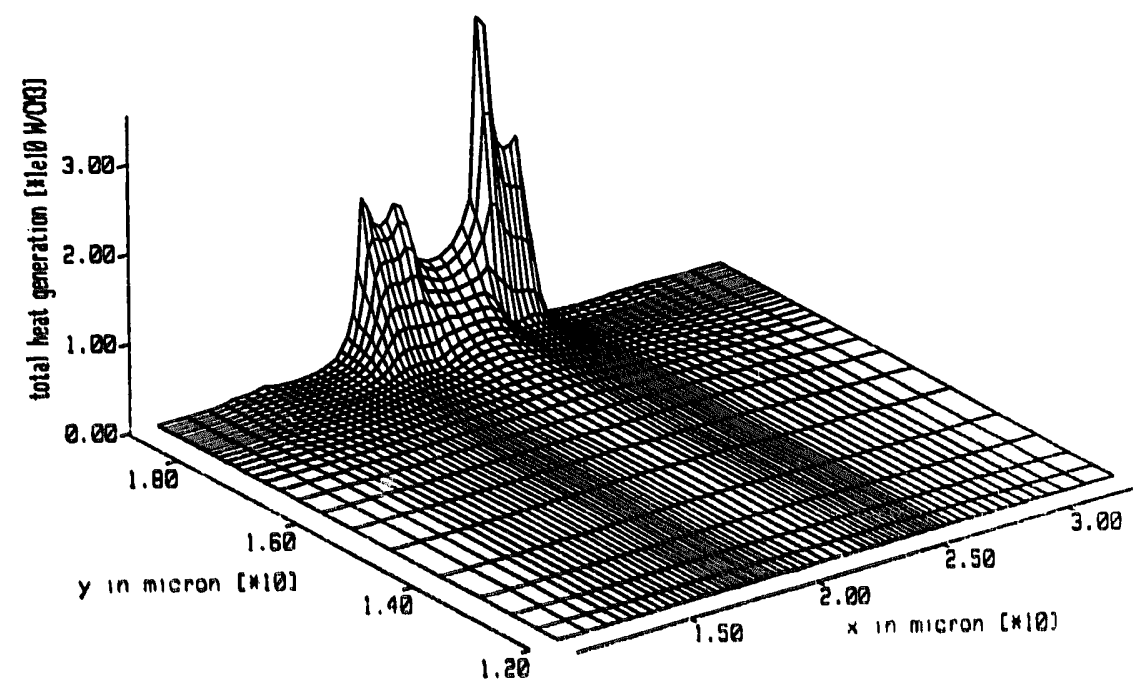

Fig. 8. Total heat generation at time $=75 \mathrm{~ns}$.

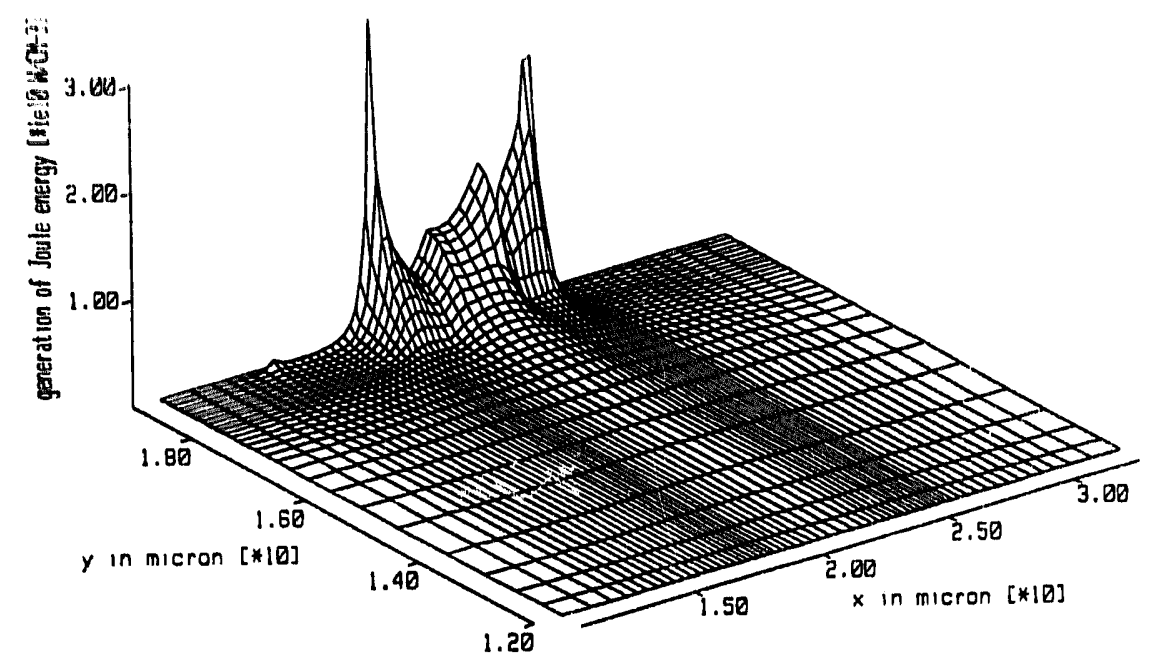

Fig. 9. Joule heat generation at time $=75$ ns. 


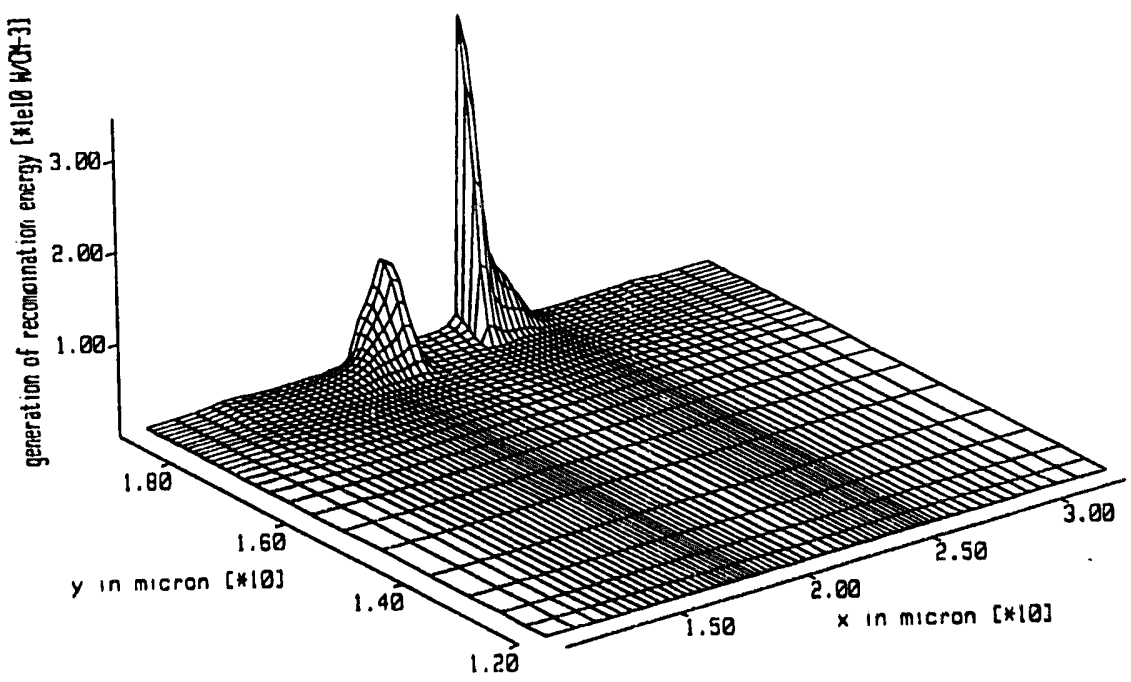

Fig. 10. Recombination heat generation at time $=75 \mathrm{~ns}$.

temperature on the recombination heat (see eqn. (16)) is not insignificant. Before the high temperatures in the device are reached the recombination heat is a much smaller fraction of the total heat generation. So the higher the temperature is, the larger the fraction of the total heat generation that is caused by recombination heat.

At the time the current reaches its maximum (at $t=10 \mathrm{~ns}$ ), the Joule heat generation is much larger than the other heat generation terms. At this time the temperatures are much lower, and the recombination heat is a much smaller fraction of the total heat generation than in the case of Fig. 10.

The Thomson heat generation comes where large gradients in the thermoelectric powers $P$ occur. $P$ is strongly dependent on the carrier concentrations $n$ and ${ }_{p}$. So where there are large gradients in the carrier concentrations Thomson heat is generated. Compared to the other terms the Thomson heat can be neglected in this case. Figure 11 shows the Thomson heat generation. Note that this heat generation term is positive in some areas and negative in others.

\subsection{Temperature distribution}

The result of the heat generation is a certain temperature distribution. Before high temperatures are reached the Joule heat generation is dominant, and determines the temperature distribution. When the current has passed its maximum and the temperature starts to rise the influence of the recombination heat starts to grow. The result is that the corner of the $n^{+}$region reaches the highest temperature just below the surface. Figure 12 shows the temperature distribution at the time the maximum temperature rise has been reached.

The thermal behaviour is much slower than the electrical behaviour. Figure 13 shows the maximum temperature in the device as a function of time. The maximum temperature (about $530 \mathrm{~K}$ ) is reached after approximately $75 \mathrm{~ns}$. 


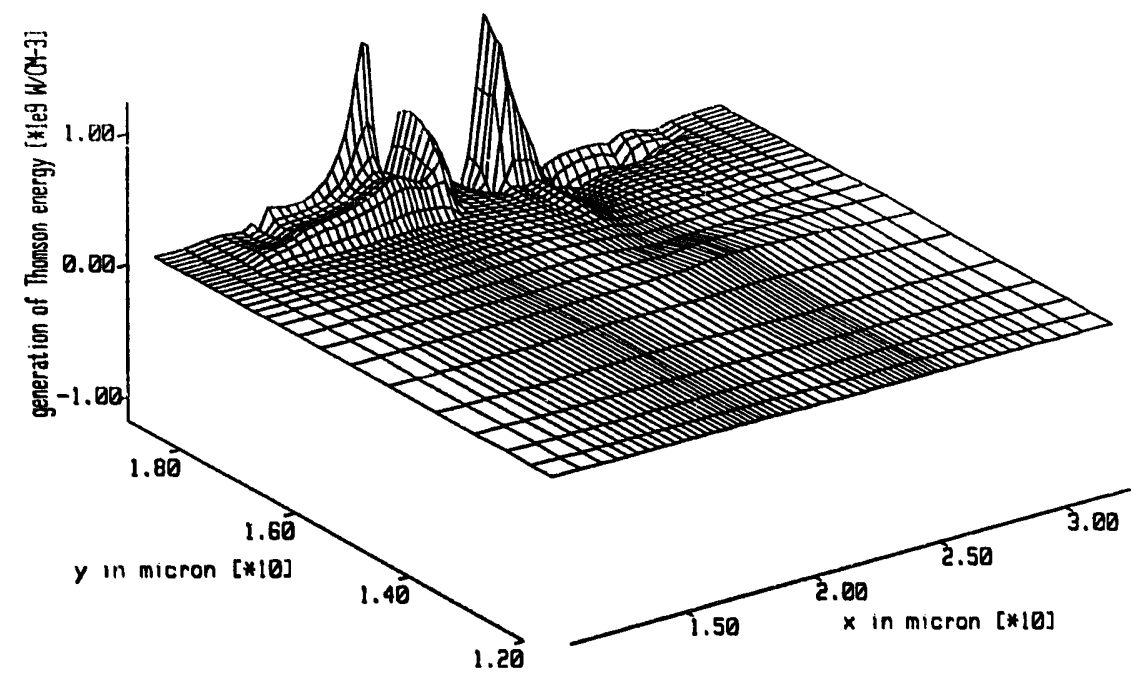

Fig. 11. Thomson heat generation at time $=75 \mathrm{~ns}$.

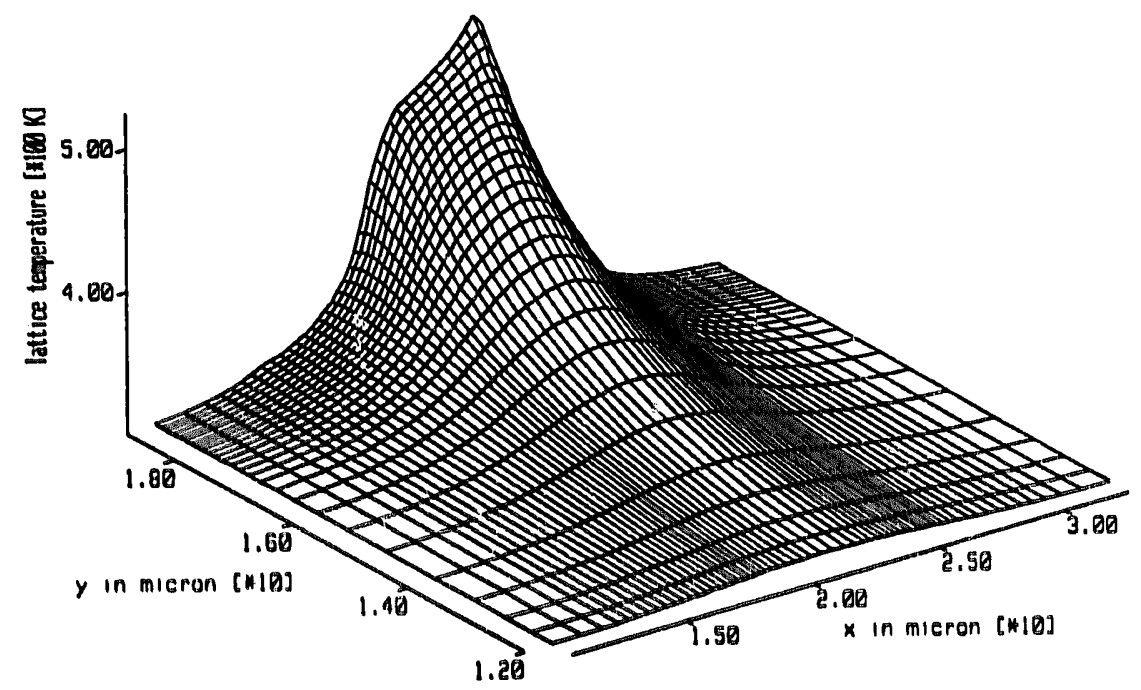

Fig. 12. Temperature distribution at ti:ne $=75 \mathrm{~ns}$.

This temperature is not likely to cause any damage to the device. Compare this figure with Fig. 3 where the maximum current through the device is reached after $10 \mathrm{~ns}$.

A few general remarks on the temperature distribution will be given next. The structure shown in the figures is only a part of the total simulated structure. In fact an extended structure has been simulated including oxide and aluminium layers, and an extension of the silicon at the left, right and lower side of the structure in Fig. 1. This is because the heat sinks (where $T=300[\mathrm{~K}]$ ) have to be located at such a distance from the heat generation region, that they do not influence the temperature distribution in the interesting time interval. As Fig. 12 shows, the heat conduction to the heat sinks has not yet influenced 


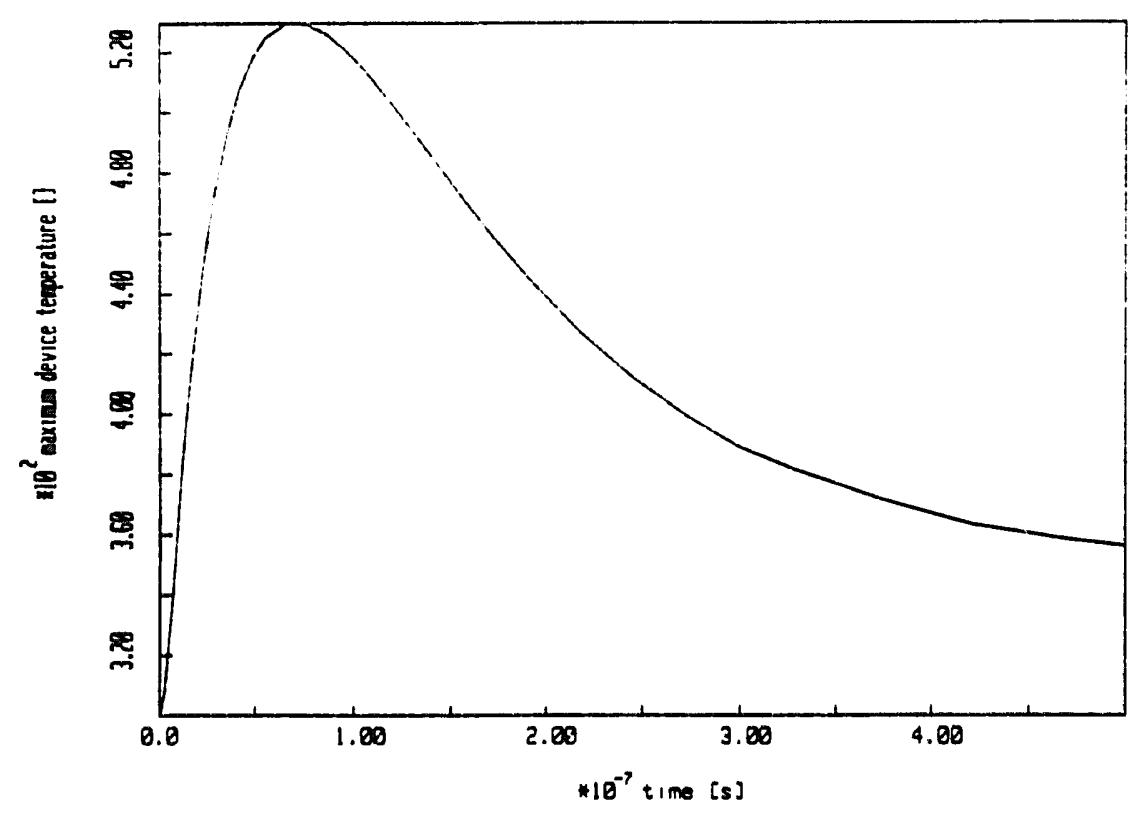

Fig. 13. Maximum device temperature versus time.

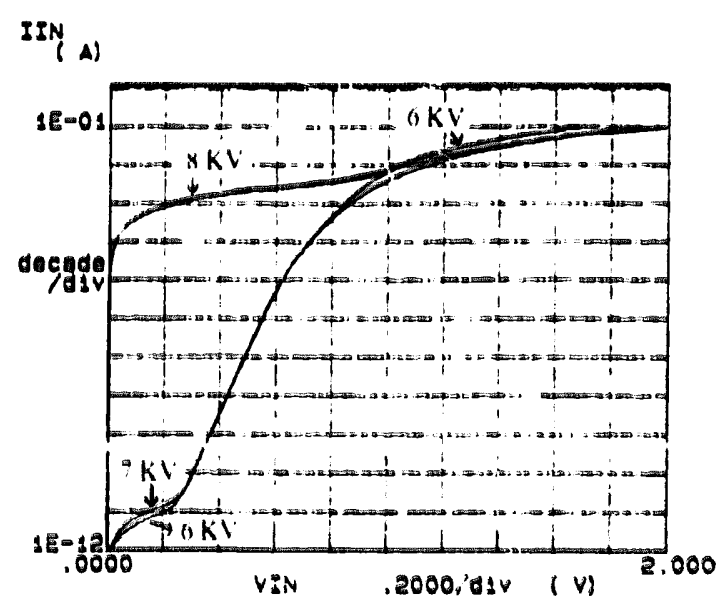

Fig. 14. Forward $I-V$ characteristic 3 of the diode without a buried layer plotted after several ESD pulses. The stress voltages after which the curve has been plotted are mentioned in the figure.

the temperature profile considerably. The heat capacity combined with the heat generation almost completely determine the temperature profile in the first $75 \mathrm{~ns}$. Heat flow has only occurred in a small area around the heat generation area. As the heat flow to the heat sinks has not really influenced the temperature distribution in the first $75 \mathrm{~ns}$, neither has the heat flow in the third dimension (that has not been simulated). Therefore temperature variations in the third dimension must be caused by variations in the heat generation (resulting from variations in the electrical behaviour). 


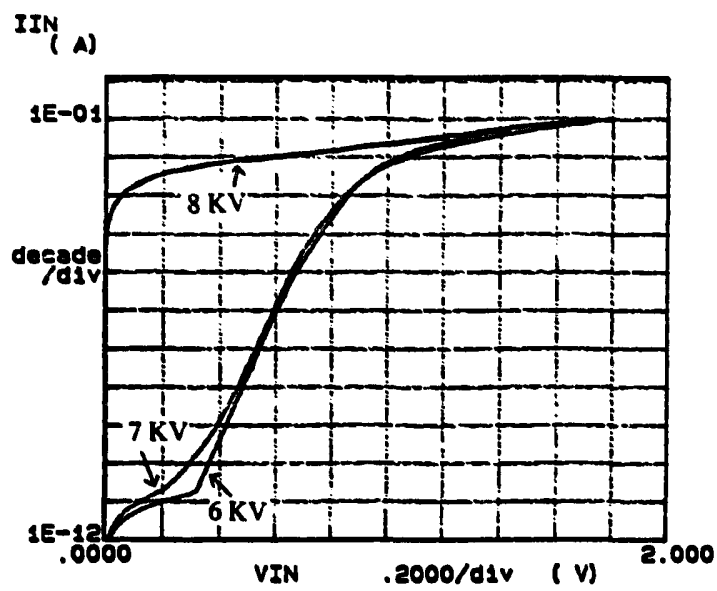

Fig. 15. Forward $I-V$ characteristics of the diode with a buried layer plotted after several ESD pulses. The stress voltages after which the curve has been plotted are mentioned in the figure.

\section{Measurement results}

The simulation results predict that for ESD pulses in forward direction the existence of a buried $n$ layer does not significantly influence the ESD susceptibility. Measurements have been performed on protection diodes with and without a buried layer. ESD pulses (HBM) were applied in forward bias on 25 $\mu \mathrm{m}$ diodes.

It has to be kept in mind that because of the finite length of the diodes three dimensional effects will be present. The $2 \mathrm{D}$ simulation results correspond with a device of infinite length, so there are no variations in the device behaviour in the third dimension. This has consequences for the temperature distribution. Regarding the temperature distribution the 2D simulation results represent the "worst case" if a homogeneous electrical behaviour is assumed in the third dimension.

After stressing the diodes the forward $I-V$ characteristics were evaluated. Figures 14 and 15 show the forward $I-V$ characteristics of the diodes without and with buried layer after pulse heights of 6,7 and $8 \mathrm{kV}$. The measurements were done with a floating bulk contact. The characteristics of the diodes before stressing were not visibly different from those after stressing with $6 \mathrm{kV}$.

\section{Conclusions}

- The models used in the device simulator may no longer be valid when very high temperatures are reached. Therefore simulation results that show very high temperatures require a careful interpretation.

- The effect of the third dimension has not been considered in the 2D simulation results presented. The electrical behaviour of the device may not be constant in the third dimension. It is expected however that the heat flow in 
the third dimension does not influence the temperature distribution considerably during the first $75 \mathrm{~ns}$. Therefore it is expected that at the time the maximum device temperature is reached, the temperature variation in the third dimension is determined mostly by the variation of the electrical behaviour in the third dimension.

- Even if the device were two dimensional, and the calculated temperature profile were correct, it is very difficult to extract the mechanism that causes degradation of the IV-curves from the simulation results.

- The previous three conclusions indicate that the application of the simulations for ESD devices is qualitative and a comparison with measurements should also be qualitative. This means that certain effects and trends can be predicted using thermo-electric device simulation but that it is difficult to exactly predict the maximum voltage (HBM) before degradation occurs.

- The simulation results indicate that the simulated ESD pulse in forward direction is not likely to cause permanent damage in the device. Measurements have confirmed this (the IV characteristics do not degrade).

- Measurements showed that both types of diodes respond to the ESD pulses in the same way. The pulse height at which the characteristics start to degrade is equal for both types of diodes. As predicted by the simulation results, the buried layer has no influence on forward ESD behaviour.

- For reverse bias ESD behaviour the absence of a buried layer will influence the characteristics. It will influence the voltage at which punch through will be reached, and when avalanche strength in the electric field is reached [8]. It will also determine the voltage at which high currents are conducted. Therefore the previous conclusion does not imply that the buried layer can be eliminated.

- Optimisation of the ESD susceptibility in forward direction by adjusting layout and doping profile can be done using the results of the ESD simulations in forward bias. The use of a symmetric structure with two $n^{+}$areas and two cathode contacts at both sides of the $p^{+}$area reduces the maximum current density by a factor of two, and the heat generation by a factor of approximately 4. The ESD susceptibility in reverse bias is more critical however. Therefore simulations should be done in reverse bias and the adjustments in doping profile and layout should be made based on the results of these reverse biased ESD simulations.

- Fully coupled thermal-electrical device simulations of ESD events in a protection diode in forward bias have been performed. These simulations can give a physical insight in discharge mechanisms and processes leading to thermal failures as a result of ESD. From the calculated temperature distributions in the device, locations with a high temperature rise that eventually lead to damage can be extracted. They can give insight in the processes that lead to permanent damage. 
- In the future, simulation of ESD in reverse bias will be investigated. The most interesting are the effects leading to hot spois and thermal breakdown.

\section{Acknowledgement}

The authors wish to thank H. van der Vlist and M. Hoeven, Philips Components Nijmegen (The Netherlands) for providing test devices and assistance with the measurements.

\section{References}

1 R.A.M. Beltman, H. van der Vlist and A.J. Mouthaan, Simulation of thermal runaway during ESD events, EOS/ESD Symposium Proceedings, 1990, 157-161.

2 N. Kusnezov and J. Smith, Modelling of EOS in silicon devices, EOS/ESD Symposium Proceedings, 1979, 132-138.

3 R.N. Rountree, C. Duvvury, T. Maki and H. Stiegler, A process tolerant input protection circuit for advanced CMOS processes, EOS/ESD Symposium Proceedings, 1988, 201-205.

4 E. van Schie, Ph. Wolbert and J. Middelhoek, TRENDY: a 2D process and device modeling and simulation environment, in Software Tools for Process, Device and Circuit Modeling, Nasecode VI Conf., 1989, 155-161.

$5 \mathrm{Ph}$. Wolbert and A.J. Mouthaan, The extension of the 2D process/device simulator TRENDY with hydrodynamic and thermodynamic device modeling, in The Numerical Analysis of Semiconductor Devices and Integrated Circuits, Nasecode VII Conf., 1991, 126-127.

6 G. Wachutka, Rigourous thermodynamic treatment of heat generation and conduction in semiconductor device modeling, IEEE Transactions on Computer-Aided Design 9 (11) (November 1990) 1141-1149.

7 R.A. Smith, Semiconductors, Second Edition, Cambridge University Press, London (1979) pp. 151-157.

8 W.J. Orvis, C.F. McConaghy, J.H. Yee, G.H. Khanaka, L.C. Martin and D.L. Lair, Modeling and testing for second breakdown phenomena, EOS/ESD Symposium Proceedings, 1983 , 108-117.

9 Ph. Wolbert, Modeling and simulation of semiconductor devices in TRENDY, Ph.D. Thesis, University of Twente, The Netherlands, 1991.

10 S. Selberherr, Analysis and Simulation of Semiconductor Devices, Springer, Wien (1984).

11 J.M. Dorkel, On electrical transport in non-isothermal semi-conductors, Solid State Electronics, 26 (8) (1983) 819-821. 